\title{
Boron nitride nanoscrolls: structure, synthesis and applications
}

Muhammad Saqib Qayyum ${ }^{1}$, Hasan Hayat ${ }^{2,3}$, Rupy K. Matharu ${ }^{5}$, Tanveer A. Tabish ${ }^{3,4}$, Mohan Edirisinghe ${ }^{5}$

${ }^{1}$ Department of Materials Science and Engineering, Norwegian University of Science and Technology, NTNU, 7491 Trondheim, Norway.

${ }^{2}$ College of Engineering, Swansea University, SA1 8EN, Swansea, United Kingdom.

${ }^{3}$ College of Engineering, Mathematics and Physical Sciences, University of Exeter, EX4 4QL, Exeter, United Kingdom.

${ }^{4}$ Centre for Graphene Science, University of Exeter, EX4 4QF, Exeter, United Kingdom

${ }^{5}$ Department of Mechanical Engineering, University College London, Torrington Place, WC1E 7JE, London, United Kingdom

\section{Abstract.}

Boron nitride nanoscrolls (BNS) are open-ended, one-dimensional (1D) nanostructures made by the process of rolling boron nitride nanosheets (BNNS) into a scroll-like morphology. BNS offer a high surface area to volume ratio and possess many unique properties (similar to carbon nanotubes (CNT), carbon nanoscrolls (CNS) and boron nitride nanotubes (BNT)) such as high resistance to oxidation, chemical stability, increased lubrication, high-temperature resistance, electrical insulation, the ability to cap molecules inside and at the ends, and a wide band gap regardless of chirality. Despite these attractive features and properties well suited for applications in biotechnology, energy storage, and electronics, the true potential of boron nitride, and BNS as the next 'miracle material' is yet to be fully explored. In this critical review, we assess, for the first time, various studies published on the formation, structural and dynamic characteristics of BNS, potential routes for BNS synthesis, and the toxicology of BNS. Finally, the future perspectives of BNS are discussed in view of its unique and exceptional candidacy for many (real-world) applications.

Key Words: boron nitride nanoscrolls, exfoliation, properties, synthesis, applications.

\section{Introduction.}

Two-dimensional (2D) nanostructures such as graphene[1] have been studied extensively over the past decade (or so) for their applications in key areas such as electronic devices[2-4], renewable energy[5], biomedical science[6-8], civil engineering[9], and water treatment[10]. However, world experts in the field of materials science claim that "the real treasure is yet to be explored"'[11]. The significance of these materials can be fathomed by the fact that the British government alone has invested up to $£ 50$ million in graphene research to date[12], and globally, this figure is expected to exceed \$1 billion by the year 2025[13]. In addition to carbon nanostructures, non-carbon 2D nanomaterials, such as boron nitride (BN), molybdenum disulphide $\left(\mathrm{MoS}_{2}\right)$, molybdenum ditelluride $\left(\mathrm{MoTe}_{2}\right)$, etc., have also demonstrated enormous potential, with state-of-the-art research leading to the exploration of over 500 non-carbon 2D materials to date[14]. Figure 1 shows a comparison of research conducted on graphene-based $2 \mathrm{D}$ materials and one of their respective isoelectric non-carbon counterparts, i.e., BN which is the main focus of this contemporary review. 


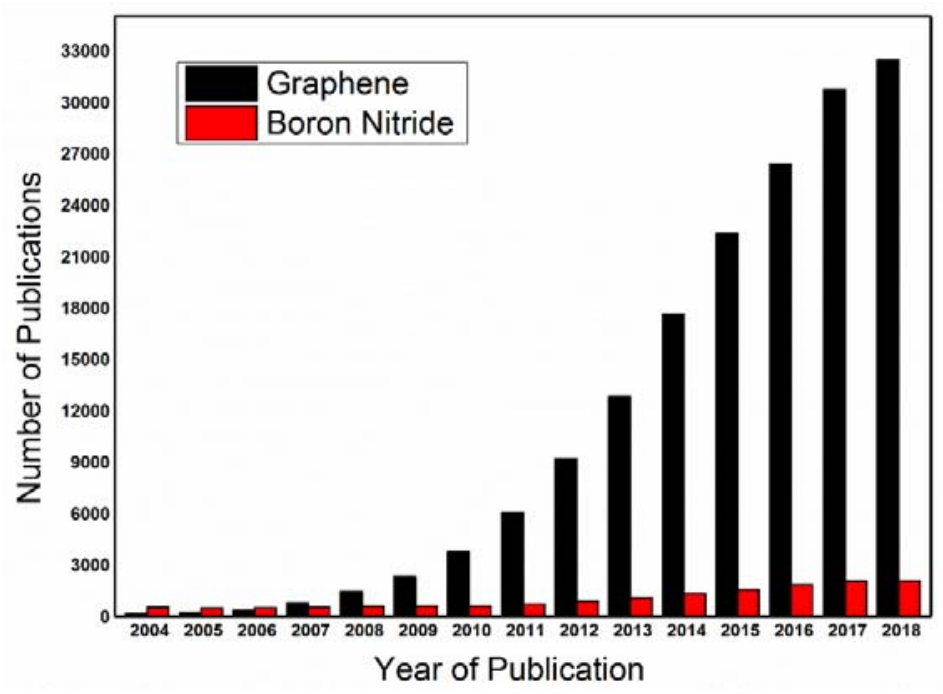

Figure 1. Graphene and BN research over the last 14 years. (Source: Web of Knowledge/keywords: graphene/boron nitride).

Hexagonal boron nitride ( $\mathrm{hBN}$ ) is a structural analog of graphene consisting of a layered structure in which strong covalent bonds bind boron and nitrogen atoms, and interplanar layers are held together by weak van der Waals forces (VDW). Boron atoms are naturally attracted to nitrogen atoms due to their higher electronegativity that typically leads to the formation of an ionic character. A band gap lies between the valence and conduction bands, and it is due to this phenomenon that $\mathrm{hBN}$ is classified as a wide band gap semiconductor with excellent thermal and chemical properties [15-17]. Moreover, it has been reported in the literature that hBN is highly resistant to oxidation [18], provides corrosion protection at elevated temperatures [19], offers good hydrogen storage capacity [20-22], and has significant potential for use in biomedical applications, such as drug delivery [23-27], neutron capture therapy [28-31], and cell imaging [32-34].

Like many of its nanomaterial counterparts, various synthesis studies on hBN have demonstrated that it can indeed exist in various dimensionalities, such as OD quantum dots [35], 1D nanotubes [36, 37], nanoribbons [38], and nanoscrolls[39, 40], 2D nanosheets [41], nanomeshes [42], and 3D nanoflowers [43]. Amongst the aforementioned dimensionalities, of utmost importance and largely underexplored are boron nitride nanoscrolls (BNS); flat boron nitride nanosheets (BNNS) rolled into a spiral-like morphology with a 1D scroll structure, and consisting of hollow cores with a unique open-ended morphology, and offering a large surface to volume area (see Figure 2a). Essentially, BNS are structural analogs of carbon nanoscrolls (CNS) [44-48], possessing exotic physiochemical properties that are strikingly dissimilar in electrical and insulating properties to CNT [49], and CNS [50-52], i.e., in contrast to CNTs, the electrical properties and band gap of BNS is independent of their chirality [53]. Also, BNS offer tunable interplanar distance by intercalation or doping making them a strong candidate for application in hydrogen storage, mass manipulation, and targeted drug delivery [54-57]. Therefore, investigating the self-scrolling of $\mathrm{BN}$ and developing multi-methods to induce the 
folding of BN are of great interest and significance for the preparation of novel structures beyond conventional material preparation techniques.

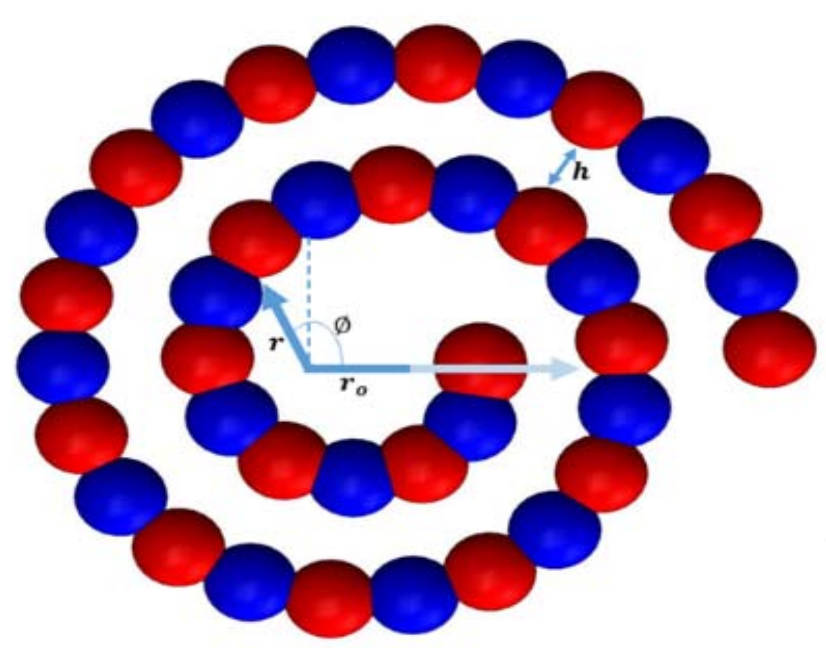

(a)

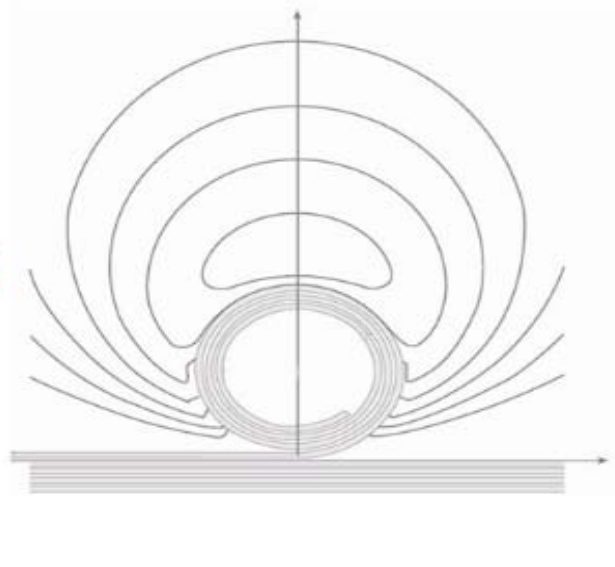

(b)

Figure 2. Schematic representation of hexagonal boron nitride nanostructures: (a) nanosheet rolled-up to form a nanoscroll with a core radius $r_{o}$, and interlayer spacing $h(b)$ streamlines around a nanoscroll in a viscous liquid. Figure (b) adapted from [58], with permission of Springer Nature.

This paper presents, for the first time, a detailed review of the recent developments and published literature related to BNS. In particular, Section 2 provides an insight into the structure, Section 3 discusses the synthesis and possible experimental routes, and Sections 4 shed light on the properties and applications and 5 considers the toxicity potential of BNS. Moreover, future directions and suggestions for BNS research have been discussed and proposed in Section 6.

\section{Structure and kinetics of BNS formation.}

Intrinsically, graphite and hBN have close bond length values with a similar honeycomb arrangement of atoms and possess similar structural and mechanical properties. In this regard, the energetics and kinetics used to describe the formation of CNS can also be used to describe the formation of BNS. It is well understood that two main contributions dominate the interlayer interaction between h-BN sheets; (1) the VDW forces for anchoring the layers at a fixed distance, and (2) the electrostatic forces dictating the optical stacking mode [59]. Here, it is worth highlighting that hBN is a wide band gap semiconductor with polar bonding $[53,60,61]$ which typically displays completely different electronic and insulating properties in terms of applications such as usage in insulation of electric packaging[62], bandgap tailoring[63], and to increase thermal stability of epoxy composites[64]. BNS derivatives can exist in lengths ranging from a few hundred nanometers up to several microns with variable diameters, and can be envisaged as rolled up nanosheets in Archimedean spirals described by the polar equation [65]: 


$$
r=r_{o}+\frac{h}{2 \pi} \emptyset
$$

where $r$ is any point that belongs to the scroll for a defined core radius $r_{o}, h$ is the interlayer spacing, $\emptyset$ has values ranging from 0 to $2 \pi N$, and $N$ is the number of turns made by the nanoscroll (Figure 2a). In the case of BNS, the scroll types are defined by an angle $\theta$; for a zigzag configuration $\theta=0$, for an armchair configuration $\theta=90^{\circ}$, and for chiral nanoscrolls $0^{\circ}<$ $\theta<90^{\circ}$.

The formation of nanoscrolls is strongly dependent on the process of synthesis of the parent material (in this case nanosheets) as this has a significant effect on the overall morphology and size distribution. To develop a better understanding of nanoscrolling, we consider multiple sheets of a layered material; in this case, hBN nanosheets or plates distributed in a liquid, consisting of double stressed layers, as shown in Figure $2 \mathrm{~b}$. The exfoliation of the external layers in this structure takes place following the intercalation of liquid between the internal layers, and the twisting of the exfoliated layer, hence resulting in the formation of a nanoscroll; this takes place in a small enough time frame as compared to the diffusion growth time. The internal elastic stresses in a nanoscroll are typically much lower when taking into account the layers that determine the energy profit of the nanoscroll twisting process[58, 66]. Perim and Galvao [67] postulated the minimum requirements for the scrolling process to be self-driven, and are as follows: there are two energy contributions required for BNS initiation and successful scrolling. 1) an elastic energy increase caused by the bending BN layer (decreasing stability), and 2) the free energy decrease generated by the VDW interaction of overlapping layers ultimately resulting in increased stability. For a nanoscroll to be in an equilibrium state and remain in that state for an indefinite period (without the energetics of returning to a nanosheet), the essential geometric parameters are the scroll width and length. This equilibrium state can be defined as $E_{s c r}=E_{\text {int }}+E_{\text {bend }}$, where $E_{\text {int }}$ is the energy arising from the interaction of the interlayers in the overlapping regions, and $E_{\text {bend }}$ is the bending energy due to the restoring force of the mechanical strain ( which is always higher than the interaction energy $\left(\left|E_{\text {int }}\right|\right)$, i.e., $E_{s c r}>0$ [68]). Although there is a critical diameter requirement of $\sim 20 \AA$ for scroll stability above which the scrolled structures are more stable than their starting material, the self-scrolling process itself is not self-initiating, and there must be a starting energy increase which must be provided by some external source, i.e., by sonication, microwave irradiation and mechanical triggering or (from a kinetic point of view) initiated by stress of either a structure or electrical in nature caused by the asymmetry of the layer [69]. The resulting nanoscrolls formed by this energy driven mechanism are more stable than the previous state (nanosheets) [70]. Siahlo et al. [71] studied the energetics of nanoscrolls using an analytical model, and predicted the initial lengths for which stable and energetically favorable nanoscrolls are possible ranging from 7 to $30 \mathrm{~nm}$ for all the nanoscroll types, and the energy barriers to rolling and unrolling of these nanoscrolls are within $10 \mathrm{eV}$. Furthermore, the authors [71] predicted the lifespan of a nanoscroll to be more than 1000 years, based on the following formula: 


$$
\left.\frac{1}{\tau}=\Omega^{\left(-{ }^{E_{2}} / k T\right.}\right)
$$

where $\Omega$ is the frequency multiplier, $k$ is the Boltmann constant, $T$ is temperature and $E_{2}$ is the difference of potential energies between the unstable and stable state of the nanoscrolls. For a stable nanoscroll, the core radius must be larger than the interlayer spacing, and the equilibrium state for the nanoscroll can be described as [72]:

$$
d W_{b}+d E_{s}=0
$$

where, $d W_{b}$ is the work change by the bending force and $d E_{s}$ is the change in binding layer energy of the nanoscroll.

\section{Synthesis.}

\subsection{Methods to synthesize BNS.}

The existence of BNS was first predicted by Perim and Galvao in 2009 [67], and a theoretical approach to the synthesis of BNS was proposed by the same group later in 2011[73]. In this method, molecular dynamics simulations were carried out to simulate BNNS deposited over a rigid $\mathrm{SiO}_{2}$ substrate and with a carbon nanotube placed over the BNNS to trigger the scrolling process. The underlying concept of this approach is based on the notion that VDW forces of interaction cause the deformation of the sheet to roll itself around the CNT, hence maximizing the contact area, and since there is no resistance due to vacuum, the scrolling process will subsequently be self-sufficient (see Figure 3a). An improved version of this process was further reported by Perim et al. [74] by utilizing a substrate with pits/chambers in place of a plain $\mathrm{SiO}_{2}$ substrate. Although a relatively efficient synthesis process, this technique presents challenges in terms of up-scaling as it is difficult to overcome the forces of interaction between the sheet and the substrate in more realistic environments under ambient conditions (see Figure 3 (b)). In another theoretical study based on molecular mechanics, BNS formation was shown to depend mainly on the size of the triggering BNNT while satisfying other requirements [75]. In this scheme, it was shown that (1) if the width (W) of the hBN nanosheet is $\leq 9.232 \AA$, a helix will form instead of a scroll, (2) if the width of the nanosheet is $>17.753 \AA$, a nanoscroll will form on the BNNT surface, and (3) if the nanosheets have widths lying between $9.232 \AA$ and $17.753 \AA$, they are deemed unstable.
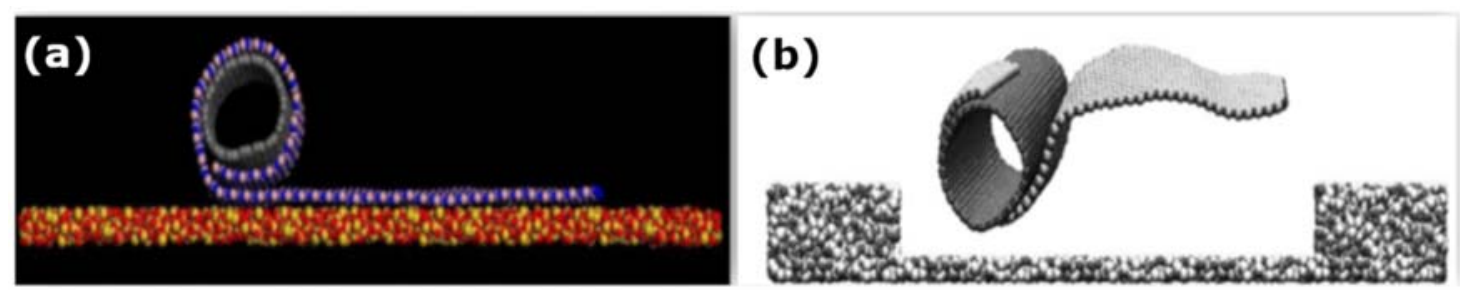

Figure 3. Proposed setup of BNS formation using (a) CNT initiated scrolling and (b) CNT initiated scrolling with pits/chambers to reduce friction. Figure 3(a) adapted with permission from [73]. Figure 3(b) reproduced from [74], with the permission of AIP Publishing. 
The first experimental study reporting the successful synthesis of BNS was published in [39] by adopting a mechanical approach via a spinning disc processor (SDP) under shear forces at ambient conditions (see Figure (4a)). In this top-down method, boron nitride flakes were suspended in N-methyl-2-pyrrolidone (NMP), and a jet feed of suspension was directed towards a disc rotating at $2500 \mathrm{rpm}$ consequently giving rise to dynamic thin films with a high surface area to volume ratio. The shear forces generated in the dynamic thin films resulted in the simultaneous peeling off of BN layers and scroll formation. The scrolls formed in this process were multilayered, consistent in length with the parent material, and had step-like rolling edges along the scroll (see Figure $4(b-c)$ ). One of the advantages of such a process is a resulting yield of $5 \%$ (depending on the particle size of the parent material) while disadvantages include non-uniformity in scrolling with some scrolls lacking an internal hollow space.

In another study, boron nitride nanosheets and nanoscrolls were synthesized using an alkali assisted molten hydroxide method [40]. The process presents advantages, such as low cost and simple (i.e. single-step method) and uses an autoclave to prepare melts of $\mathrm{KOH}$ and $\mathrm{NaOH}$ at $180{ }^{\circ} \mathrm{C}$ for 2 hours, i.e. the intercalation caused by $\mathrm{K}^{+}, \mathrm{Na}^{+}$and $\mathrm{OH}^{-}$ions causes exfoliation of bulk hBN (see figure 4(d)). The author demonstrated that nanosheets and nanoscrolls of varying sizes (Figure $4(\mathrm{e}-\mathrm{f})$ ) were produced from this process. In addition, the products can disperse in a variety of solvents, and the process can be applied to other layered materials. However, there is a significant amount of unreacted hBN, defect formation, and impurities during this synthesis process which require extensive cleaning strategies. Other drawbacks of this process include having no control over the size and diameter of nanoscrolls, and the overall yield of this process being substantially low (nanosheets and nanoscrolls @0.191\%).
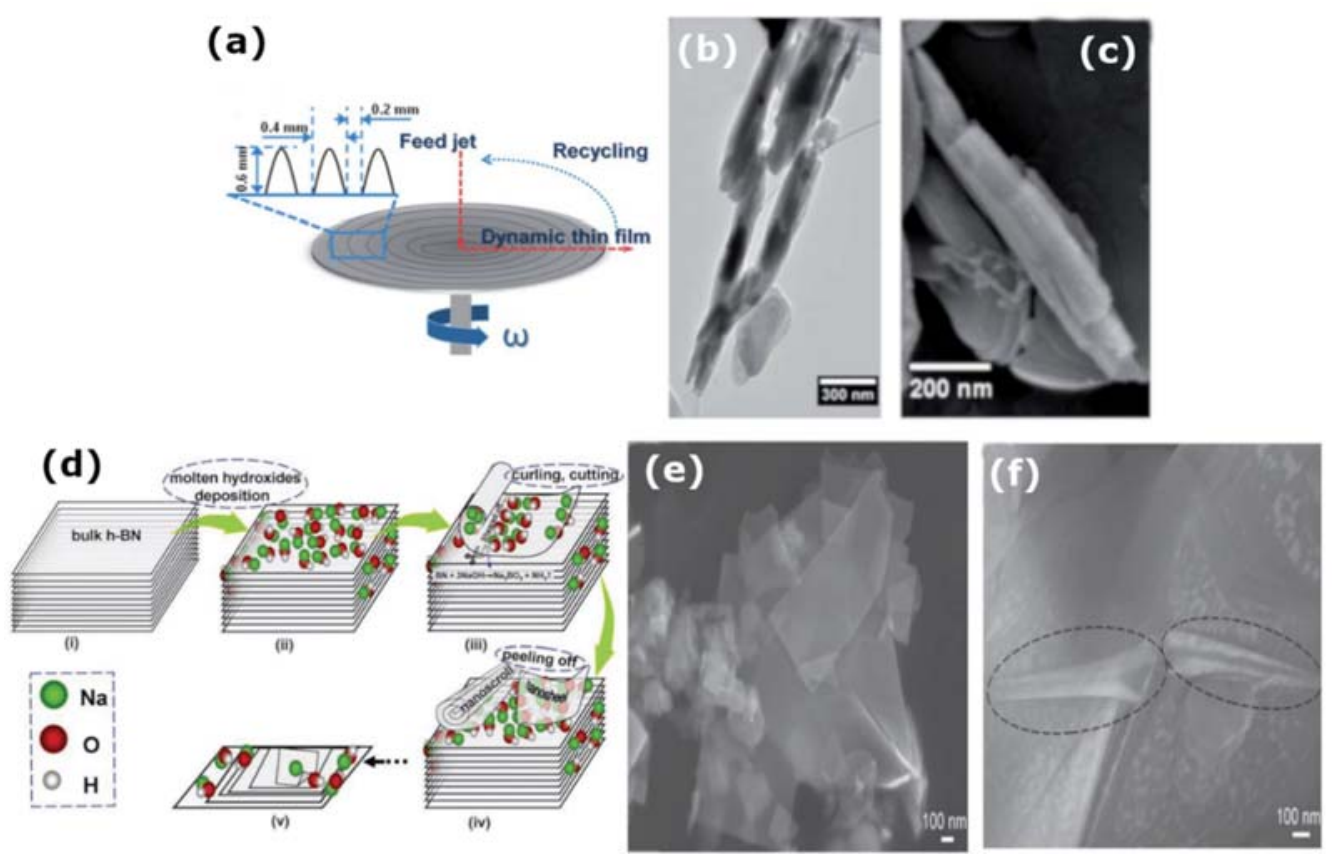

Figure 4. Experimental methods for BNS synthesized via exfoliation techniques. (a) Schematic of the spinning disc processor. (b-c) Scanning electron microscopy (SEM) images of BN nanoscrolls. (a-b) adapted from ref. [39], 
with permission from The Royal Society of Chemistry. (d) Schematics of nanosheets and nanoscrolls synthesized using intercalation of molten alkali salts. (e-f) SEM images of typically curved nanosheets several hundred nanometers in size (circled in black). (d-f) adapted with permission from ref [40], copyright 2013, John Wiley and Sons.

Suh [76] presented an innovative approach to synthesize hBN nanoscrolls based on a density gradient method. Firstly, BNNS were prepared by homogenizing BN powder with orthodichlorobenzene (ODCB) followed by heating ODCB with $\mathrm{N}$-(2-(aminoethyl)-3 $\alpha$-hydroxy-5 $\beta$ cholan-24-amide) (LCA), and then combining the end product with the initially formed BNNS resulting in precipitates of LCA induced BNS. Nanoscrolls of varying diameters were obtained using this approach and grouped into two types (small; 20-60 nm, and large; $300 \mathrm{~nm}$ up to a few micrometers). The increase in the size of nanoscrolls depends on the growth of the LCA fiber, and by carefully increasing the amounts of LCA (see Figure 5(a-b)); hollow core BNS are obtained by dissolving LCAs in methanol and subsequent washing. Although this relatively straightforward process enables the use of template-based growth requiring only the use of chemicals, the yield via this process is not easy to determine. Also, excessive washing must be carried out for the complete removal of LCAs from BNS. The density gradient method adopted by Suh's group reported in [54] was further used to form BNS-NPs nanocomposites (see figure 5(d)) using hBN templates [55]. In a multi-step method, magnetic NP solutions of $\mathrm{Fe}_{3} \mathrm{O}_{4}$ and $\mathrm{Au} @ \mathrm{Fe}_{3} \mathrm{O}_{4}$ were mixed with exfoliated boron nitride nanosheets and sonicated for 30 mins. BNS- $\mathrm{Fe}_{3} \mathrm{O}_{4}$ and $\mathrm{BNS}-\mathrm{Au} @ \mathrm{Fe}_{3} \mathrm{O}_{4}$ formation proceeded by treating the purified h-BN - NP sheet solutions (NPs loaded onto hBN nanosheets) in ODCB with a hot solution of LCA in ODCB

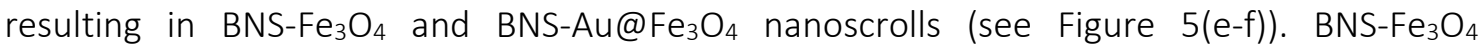
nanoscrolls had diameters ranging from $60-80$ nm and BNS-Au@ $\mathrm{Fe}_{3} \mathrm{O}_{4}$ with diameters of 10 $\mathrm{nm}$, respectively. Moreover, the authors of this research claim that nanoscrolls with high yield can be formed by carefully increasing the amount of LCA fibers. 

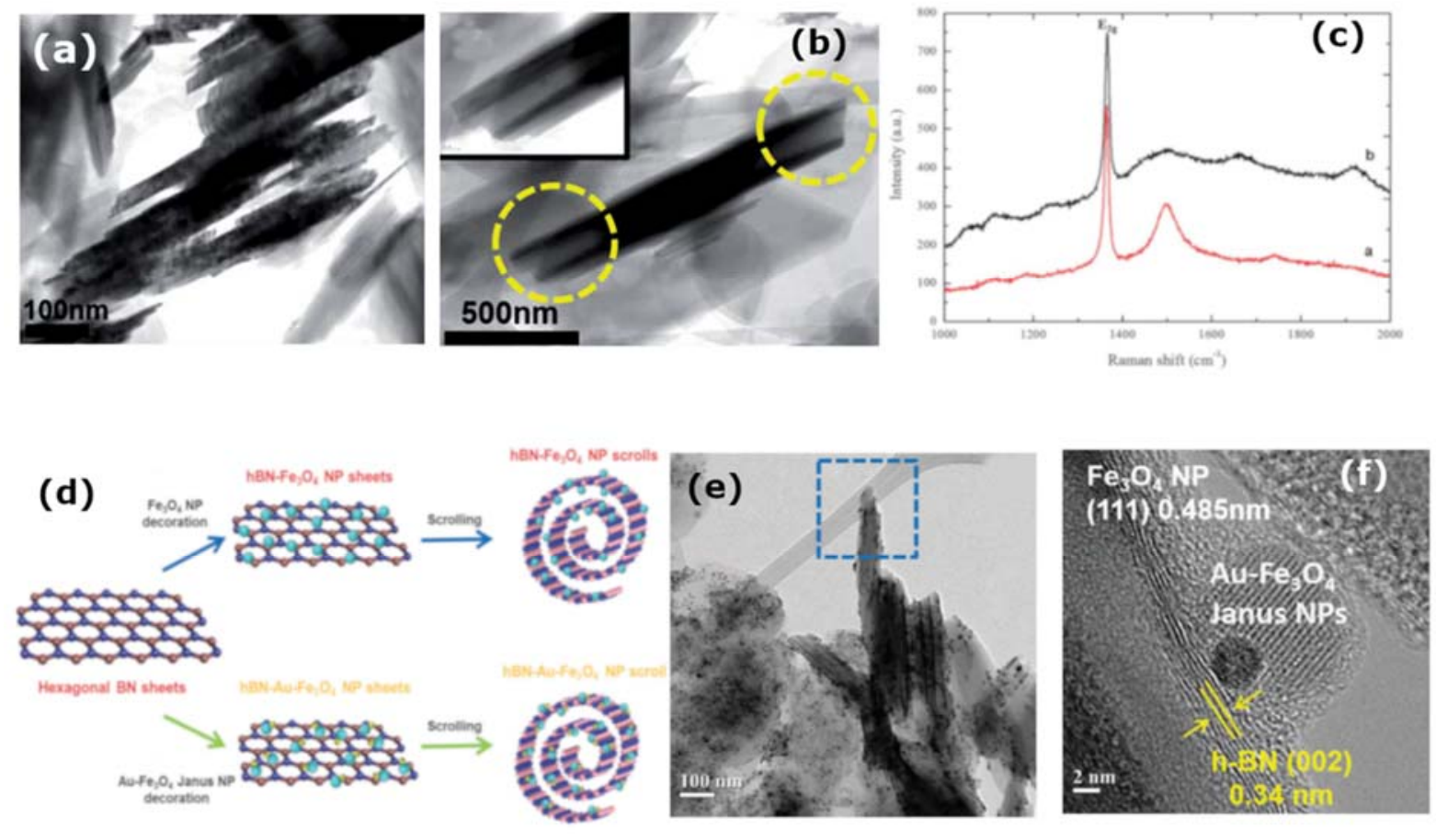

Figure 5. Experimental methods for BNS synthesis via template based techniques. (a-b) TEM image of BNS produced from (a) LCAs and the exfoliated boron nitride sheets. (b) Small-BNS with partial dissolution of LCA inclusion at both end points. (c) Raman spectra of the exfoliated h-BN (red) and BNS at E2g phonon mode (black). (a-c) Adapted from ref. [76], with permission of The Royal Society of Chemistry. (d) Schematics of the Magnetic BN - nanocomposites. (e) Low-magnification TEM image of the synthesized h-BN-Au@Fe3O4 nanoscrolls. (f) HR-TEM image of $\mathrm{Au} @ \mathrm{Fe}_{3} \mathrm{O}_{4}$ Janus NPs with corresponding lattice spacing. (d-e) [77], adapted by permission of the PCCP Owner Societies.

\subsection{Strategies for Future BNS synthesis}

As discussed in Section 3.1, various methods have been adopted for the synthesis of BN and its derivatives. The purpose of this section is to propose possible BNS synthesis methods which have shown success with other layered materials. Based on the available literature, the following sub-sections propose various possible experimental routes for the synthesis of BNS. Table 1 summarizes the advantages / disadvantages of these experimental routes.

\subsubsection{Physical Approaches}

\subsubsection{Micromechanical Exfoliation}

Akin to graphite, BN has weak bonding in the direction perpendicular to the plane of the sheet which is caused by $\pi$ orbitals perpendicular to the plane of the BN sheet surfacing due to Van der Waals interaction and ultimately leading to BN stacking. Exfoliation, however, involves the peeling of individual layers one by one which is typically accomplished by an external force; the minimum force required to extract a single layer from the bulk must be higher than the corresponding Van der Waals force to peel off the layers successfully. Therefore, employing the scotch tape method to exfoliate BN sheets from powder and subsequently using sonication techniques can be a good starting point for fundamental research on BNS. A modified form of 
the scotch tape method presented by Yuan et al. in [78] to exfoliate two-dimensional materials could potentially be used for BNS exfoliation followed by sonication to induce scrolling.

\subsubsection{Ultrasonic Exfoliation}

Solvent-phase exfoliation of bulk BN can obtain single and multi-layer BNS in a highly polar solvent medium, such as N-methylpyrrolidone (NMP) $[79,80]$ and dimethylformamide (DMF) [81]. However, it is essential that the energy required to exfoliate BN must be comparable to the solvent-BN interaction for exfoliation to occur first followed by scroll initiation later.

\subsubsection{Shock Cooling Exfoliation}

Similar to another study by Fan et al. [82], BNS can potentially be formed by a shock cooling exfoliation method. Firstly, BN powder is sonicated in water for one hour followed by centrifuging the solution to obtain flakes of smaller thickness. Once the flakes are formed, the BN colloidal solution can be rapidly cooled by surrounding liquid nitrogen, which in turn begins the formation of ice crystals during the shock-cooling step in liquid nitrogen and acts as the driving force for the scrolling.

\subsubsection{Chemical Approaches}

The ultrasonication and mechanical exfoliation of bulk BN can produce the highest quality of BNS. However, these techniques pose certain limitations such as lower productivity and large time frames required to produce adequate quantities of nanoscale materials.

\subsubsection{Intercalation and Ultrasonication}

The process of carbon nanoscroll formation using alkali metal intercalation [83] followed by subsequent sonication can potentially lead to the formation of high-quality BNS. Attributed to its layered structure, BN is readily intercalated by alkali metals such as $\mathrm{Li}^{+}[84,85], \mathrm{Na}^{+}[86]$, and $\mathrm{K}^{+}$[87] ions, and therefore, potassium intercalation of hBN powder under ambient conditions at $200^{\circ} \mathrm{C}$ overnight can induce a mild relaxation in BN interlayers after the addition of ethanol. A possible highly exothermic chemical reaction can potentially cause exfoliation based on Eq. (4) below, and upon sonication, the exfoliated hBN sheets can curl themselves to form BNS.

$$
\mathrm{KBN}+\mathrm{CH}_{3} \mathrm{CH}_{2} \mathrm{OH} \rightarrow \mathrm{BN}+\mathrm{KOCH}_{2} \mathrm{CH}_{3}+\frac{1}{2} \mathrm{H}_{2}
$$

\subsubsection{Chemical Vapour Deposition (CVD)}

A CVD route based on $\mathrm{Fe}_{3} \mathrm{O}_{4}$ nanoparticles as a catalyst precursor was proposed by Chen et al. to synthesize CNS in [88]. BNS nanoscrolls can potentially be formed in a similar way where deposition techniques such as CVD, PECVD, ALD and, PLD could be used to grow hBN over metal oxide nanoparticles which can in turn act as a catalyst. The precursor can be deposited over a silicon wafer and hBN could be grown over the reduced FeO by adjusting the stoichiometry of the BN source. The precursor gas can be a compound of diborane + ammonia, 
and borazine $[89,90]$, and this process can become feasible for mass production of BNS with controlled size variation.

Table 1. The advantages and disadvantages of different BNS synthesis methods.

\begin{tabular}{|c|c|c|}
\hline Method & Advantages & Challenges \\
\hline $\begin{array}{l}\text { Micromechanical } \\
\text { exfoliation }\end{array}$ & Cheap, excellent quality & $\begin{array}{l}\text { Low yield, limited to fundamental } \\
\text { research. }\end{array}$ \\
\hline Ultrasonic exfoliation & $\begin{array}{l}\text { Cheap, medium quality. } \\
\text { Limited yield. }\end{array}$ & Limited size control. \\
\hline $\begin{array}{l}\text { Shock cooling } \\
\text { exfoliation }\end{array}$ & $\begin{array}{l}\text { Relatively cheap, no } \\
\text { inclusion. }\end{array}$ & Limited yield, difficult to reproduce. \\
\hline $\begin{array}{l}\text { Intercalation and } \\
\text { ultrasonication }\end{array}$ & $\begin{array}{c}\text { Relatively cheap, } \\
\text { can Functionalize BN, } \\
\text { limited yield. }\end{array}$ & $\begin{array}{l}\text { Difficult to remove impurities. may } \\
\text { change the BN structure. }\end{array}$ \\
\hline $\begin{array}{l}\text { Chemical Vapour } \\
\text { Deposition }\end{array}$ & $\begin{array}{l}\text { Scalable, good quality, } \\
\text { control over size. }\end{array}$ & $\begin{array}{c}\text { Costly equipment, substrate based } \\
\text { method. }\end{array}$ \\
\hline
\end{tabular}

\section{Properties and Applications.}

To develop an understanding of the properties of BNS requires consideration of not only their chemical properties and morphology but also critical size distribution parameters, such as the length, external and internal diameter. In this regard, 1D-hBN nanoscrolls (essentially inherited from 2D hBN) possess various unique properties, including excellent stability, high thermal conductivity, electrical insulation, and high elastic modulus, which make them promising for a plethora of applications. However, the limited studies conducted on BN nanoscrolls so far hinder the exploration of their actual properties. Here, we consider the properties of BN nanostructures closely related to BNS and the properties of the basic building block of BNS, i.e., BNNS.

It is well known that defect-free graphene is currently the strongest material, and in this regard, the structural similarities of BNNS, such as high Young's modulus, and thermal conductivity[91], make them as strong as (and in some cases stronger than) other 2D materials. It was shown in [92], that the plane stiffness of monolayer graphene and BNNS are 267 and $335 \mathrm{Nm}^{-1}$ respectively, and therefore can be used as suitable fillers in polymer composites [93]. Building on these findings, it is highly likely that BNS would show an increased stiffness owing to their rolled morphology and structural stability. Interestingly, BNSs originate from BNNS which have already shown an elastic modulus $>18000 \mathrm{~m}^{-1}$, a Young's modulus of $1.16 \pm 0.1 \mathrm{TPa}$, and excellent thermal properties with a thermal conductivity of $600 \mathrm{Wm}^{-1} \mathrm{~K}^{-1}$ [94-96]. In a molecular dynamics study by Woellner et al [97] CNS and BNS were made to strike against solid surfaces at high velocities, and it was observed that CNS and BNS behaved differently at different velocities, a factor related to their interlayer interaction [98]. Two types of impact cases were considered in [97], and it was observed that in the case of a lateral collision the kinetic energy of the striking nanoscroll below a threshold lead to partial or complete 
unscrolling and, on the contrary, for higher kinetic energies the impacts lead to a complete fracture of the nanoscrolls. For vertical collisions however, fractures are observed even at lower velocities owing to the concentration of stresses in smaller areas and the brittle nature of BN.

It is evident from our discussions so far on the structure, synthesis, and properties of BN nanoscrolls that these nanostructures present huge potential for a wide range of real-world applications. Porous nanoscroll structures offer huge potential in environmental applications, particularly when used for water cleaning [99]. These porous boron carbon nitride nanoscrolls (see figure 6(a)) maintain a very high BET surface area of $890 \mathrm{~m}^{2} \mathrm{~g}^{-1}$ with micropores of $1.4 \mathrm{~nm}$ and mesopores of $20 \mathrm{~nm}$ width and can retain up to $93 \%$ of absorption capacity after regeneration, making them strong candidates for water treatment applications (see figure $6(b))$. When used for industrially polluted water treatment these nanostructures were able to efficiently clean dyes of methylene blue and congo red showing excellent absorption capacities of $250 \mathrm{mg} \mathrm{g}^{-1}$ and $620 \mathrm{mg} \mathrm{g}^{-1}$ respectively, hence adding further evidence to this proposition.

The power conversion efficiency of solar cells can be enhanced by using nanoscrolls of hBN with other 2D materials (by combining spirals of hBN, graphene, and TMDCs), and this can be used to construct novel 3D photovoltaic absorbers 6(c-d). These spiral solar cell devices can be optimized by tuning the hBN thickness to maximize the optical absorption up to $90 \%$ or the absorption relative to the number of photoactive materials used, achieving an enhancement of $762 \%$ [100]. Similarly, the high surface to volume ratio in BNS makes them a suitable candidate for energy storage applications, such as hydrogen storage. The dipolar nature of B$\mathrm{N}$ bonds in BNS can lead to stronger absorption of hydrogen whereas the chemical and thermal stability would ultimately result in a stable, lightweight hydrogen gas accumulator. With regards to surface area(which is one of the crucial factors in determining hydrogen storage capacity), BNNTs have shown a high hydrogen storage capacity of $3.0 \mathrm{wt} . \%$ at $298 \mathrm{~K}$, and 100 bar hydrogen pressure [101]. It can be envisaged from these findings that the higher specific surface area would ultimately result in an increased hydrogen storage capacity which is ideal for energy storage applications.

In addition to biomedical, water treatment and energy storage applications, BNS also have massive potential for use in modern day electronic devices. Xie et al. [46] fabricated a carbon nanoscroll (CNS) based electronic device by placing the nanoscroll between two metallic contacts over a silicon substrate, and demonstrated that the CNS was able to withstand a current density of up to $5 \times 10^{7} \mathrm{~A} / \mathrm{cm}^{2}$. Li et al. [102] further demonstrated via a theoretical study on the quantum electronic transport of carbon-based nanoscrolls that the conductance depends strongly on not only the nanoscroll radius but the temperature as well. It can be deduced from these studies that nanoscrolls are potentially well suited for electronic applications especially when compared to their traditional and nanotube counterparts. One of the main advantages nanoscrolls present over nanotubes lies in the ability of nanoscrolls to carry current through each of their layers as opposed to nanotubes (which only carry current 
through their outermost layer), and it is this property which makes nanoscrolls highly suitable for potential use as circuit interconnects in modern day electronic devices.
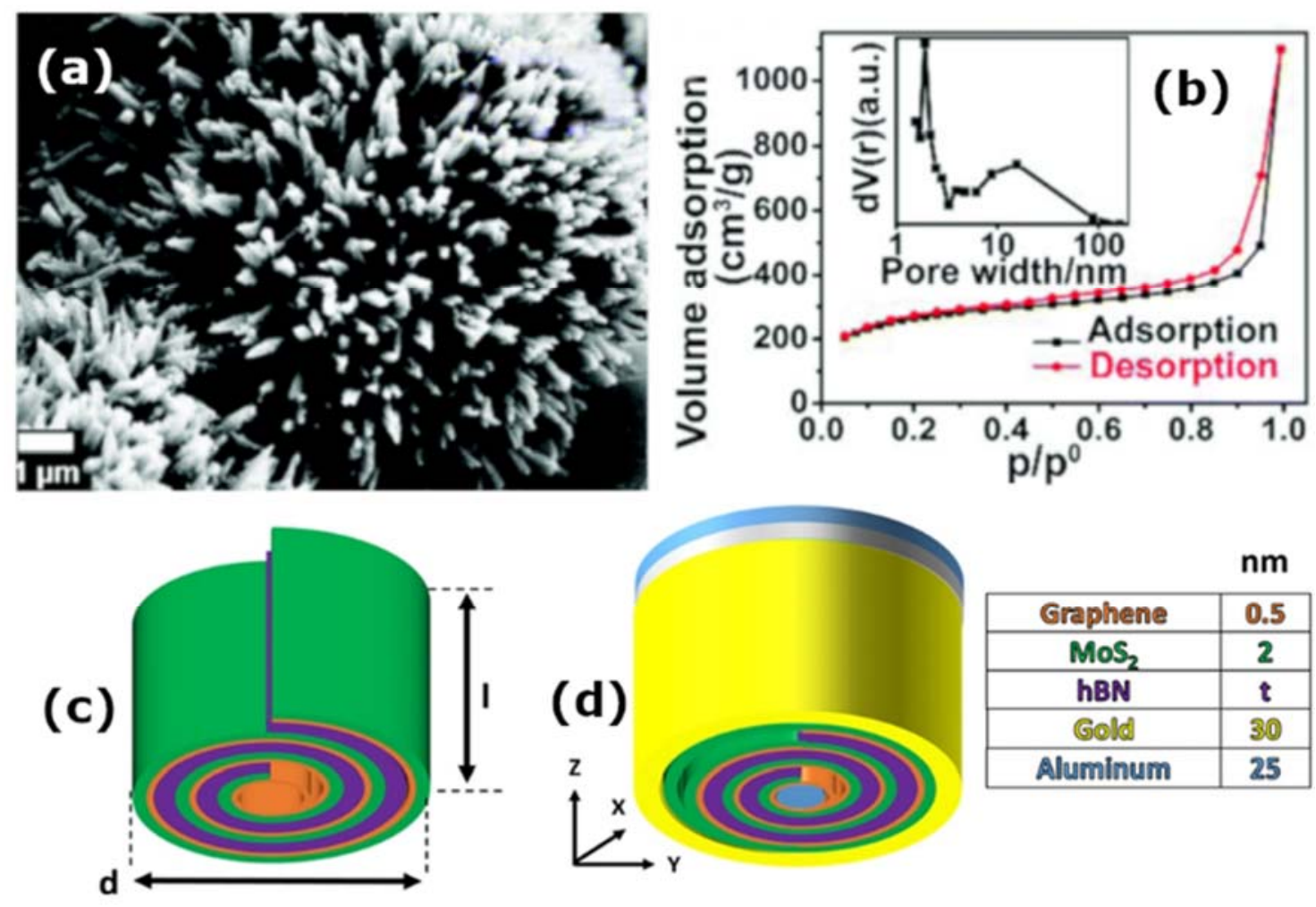

\begin{tabular}{|c|c|}
\multicolumn{1}{c}{} & \multicolumn{1}{c}{$\mathbf{n m}$} \\
\hline Graphene & $\mathbf{0 . 5}$ \\
\hline MoS $_{2}$ & $\mathbf{2}$ \\
\hline hBN & $\mathbf{t}$ \\
\hline Gold & 30 \\
\hline Aluminum & 25 \\
\hline
\end{tabular}

Figure 6. Applications of BN nanoscrolls. (a) SEM image of BCN nanoscrolls. (b) Nitrogen adsorption/desorption isotherms. The inset shows corresponding pore size distributions. Adapted from ref. [99] with permission of The Royal Society of Chemistry. (c) Schematic of 'spiral cell structure'; parameters, d, l, and t stand for diameter of the roll, length of the cylindrical structure, and thickness of the hBN layer. (d) 'Core-shell structure' of the spiral solar cell. Back reflector is connected to the core contact. Gold (aluminum) is chosen as a shell (core) selective contact. Adapted from ref. [100] @ IOP Publishing.

Finally, it is worth comparing the stability of BNS with its nanomaterial counterparts. As BNS originates from BNNS, they inherit the excellent thermal and chemical resistive properties from their parent material discussed in previous sections. However, it should be kept in mind that BNS stability is size and chirality dependent with all scrolls obeying a critical internal diameter limit. Therefore, once properly scrolled, BNS are considerably stable over a very long period of time and even more stable than their parent material i.e. BNNS. Table 2 summarizes various literature published on the stability of BNS in comparison to other nanomaterials, i.e. CNS, CNT and BNT. 
Table 2. Comparison of the stability of BNS with other nanomaterials.

\begin{tabular}{|c|c|c|}
\hline Materials & Stability & Comments \\
\hline BNS(Theoretical) & $\begin{array}{l}\text { Armchair BNS are most } \\
\text { stable. Zigzag BNS are } \\
\text { metastable and Chiral BNS } \\
\text { are unstable[67]. }\end{array}$ & $\begin{array}{l}\text { A critical diameter for scroll stability } \\
\text { exists; beyond this limit the scrolls can } \\
\text { be even more stable than its parent } \\
\text { planar structures. }\end{array}$ \\
\hline BNS(Experimental) & $\begin{array}{l}\text { Very stable, } 20 \text { months in } \\
\text { ethanol [40]. Stable with } \\
\text { slight reduction in weight up } \\
\text { to } 800^{\circ} \mathrm{C}[76] .\end{array}$ & $\begin{array}{l}\text { Stability also depends on method of } \\
\text { production and target solvent.[40] } \\
\text { Thermogravimteric analysis (TGA) } \\
\text { studies [76]. }\end{array}$ \\
\hline CNS & $\begin{array}{l}\text { Stable ambipolar behavior in } \\
\text { both air and nitrogen [103]. }\end{array}$ & $\begin{array}{l}\text { Does not mention a stability period } \\
\text { [103]. }\end{array}$ \\
\hline CNT & $\begin{array}{l}\text { Very Stable homogeneous } \\
\text { dispersions of CNT in sodium } \\
\text { dodecyl sulfate (SDS) [104]. } \\
\text { Stable in air up to } 500 \\
{ }^{\circ} \mathrm{C}[105] .\end{array}$ & $\begin{array}{l}\text { UV-vis measurements used to } \\
\text { quantitatively characterize colloidal } \\
\text { stability of CNT. The supernatant CNT } \\
\text { concentration only falls } 15 \% \text { after } 500 \\
\text { h [104]. }\end{array}$ \\
\hline BNT & $\begin{array}{l}\text { Oxidation temperature of } \\
800{ }^{\circ} \mathrm{C}[106] \text {. Stable in air up } \\
\text { to } 1100{ }^{\circ} \mathrm{C} \text {.[105] Chemically } \\
\text { inert and structurally stable } \\
\text { [107]. }\end{array}$ & $\begin{array}{l}\text { Shows higher stability than CNT at } \\
\text { elevated temperatures. }\end{array}$ \\
\hline
\end{tabular}

\section{Toxic Potential of BNS}

BNS is an emerging graphene-related material that has attained significant attention owing to its excellent features and superior physiochemical, electrical, chemical, and mechanical characteristics, as discussed in earlier sections. As graphene has widely been explored for environmental and biological applications, therefore, its toxic potential and biocompatibility and environmental availability (and adverse effects) have widely been investigated in recent years.

Chen et al. [108] reported the first study on the toxicity of BN nanostructured materials in 2009 which revealed that BN nanotubes could deliver DNA oligomers to the interior of cells with no apparent toxicity, and suggested that BN nanotubes are more suitable for use as biological probes in biological systems in comparison to CNTs. In another study, Horváth et al. [109] studied the toxic impact of BN nanotubes against different cell types (HEK293, A549, 3T3-L1, and RAW 264.7 cells) by comparing them with pristine and functionalized carbon nanotubes, and found that these nanotubes are more toxic than carbon nanotubes. With the increasing exposure of different forms of boron nitride, it is obligatory to explore the biological responses 
of boron nitride, in dose, and in time-dependent manners. The absence of toxicity studies on BNS warrants the realization of its commercial and real-world applications, and as the number of applications of BNS grows, occupational exposures will most likely increase. Recent reports on in-vitro toxic effects of different derivatives of BN seem to contradict each other [109-113]. Synthesis routes, chemical reagents, precursors, particle size, surface charge, size distribution, sharp edges of scrolls and the two-dimensional nature of BN induce adverse effects in biological systems $[114,115]$, and it is crucial that these parameters are taken into account when assessing the bioavailability and biocompatibility of BNS. In general, such emerging materials should be investigated in in-vivo toxicity models over chronic levels. The generation of reactive oxygen and nitrogen species has not been evaluated, given that generation of these oxygen/nitrogen-centered species induce inflammation as a result of the loss of primary and secondary anti-oxidants [116-118]. Furthermore, administration routes of inhalation in animal models need to be investigated since human exposure to various nanomaterials is most likely to occur through inhalation and these nanomaterials arise in the form of aerosols and colloidal suspensions[119]. Such interactions of BNS with living systems can play a vital role in the understanding of the toxicity of nanomaterials and determine their fate to solve real-world problems.

Its potential for use as an antimicrobial agent is yet to be fully explored. Among the excellent properties BNS possess, their effect on microbial growth or survival remain largely unknown, although it may be related to colonic bacterial metabolism for the future biomedical application of these emerging 2D miracle nanostructures. Furthermore, it may be possible to exploit the properties of BNS much like we have begun to utilise graphene nanoplatelets in antimicrobial work [120]. Here it was demonstrated that graphene nanoplatelet-loaded polymeric fibres produced by novel pressurised gyration [121] showed significant antimicrobial potential when the graphene nanoplatelet loading was increased to $8 \mathrm{wt} \%$. The surface of the fibres with protrusions of graphene nanoplatelets played a key role, also hydrophobic $\pi$ regions and functional groups, including carboxylic and hydroxyl groups at the surface of graphene improves its interactions via hydrophobic and electrostatic mechanisms [122]. BNS and its analogues may be incorporated on the surface of the fibres in a similar way and may will extend the family of nanocomposites as antimicrobial agents [123].

\section{Summary and Future Perspectives.}

Ever since the groundbreaking and Nobel prize-winning discovery of graphene in 2004, research on graphene and graphene-based 2D materials has increased exponentially with an estimated average of more than 40 academic papers published a day in this field [124]. However, more recently the trend has shifted to other 2D and 1D materials out of which hexagonal boron nitride holds exceptional potential for a wide range of exciting applications, as discussed in previous sections. Due to the difficulty in separating hBN layers (without compromising quality and quantity), there has been a lack of research on how BN compares to graphene, especially with regards to nanoscrolls, and the few methods that have been published suggest sonication, intercalation, and template-based techniques for synthesis. 
Triggering of a CNT based BNS is more appropriate for proof-of-principle research activities but scaling up is challenging with a requirement for an elaborate experimental setup. Nanoscrolls produced via intercalation would ultimately cause defects, a significant amount of unreacted media, and unwanted impurities produced which would require time-consuming cleaning procedures and may ultimately affect the overall quality and yield of the end product. In addition, peeling off BN layers to directly from nanoscrolls via mechanical procedures would induce metallic impurities from the spinning disc, and subsequently, affect achieving overall control and uniformity. A template-based approach seems to be a relatively useful method to synthesize scrolls efficiently but removing the fiber cores would be a cumbersome task and will again limit the formation of defect-free scrolls. Moreover, there is still a need for advanced research that can enable the availability of efficient methods for mass production of defectfree nanoscrolls. Altogether, there is currently a lack of studies indicating the direct or indirect application of BNS. In such a scenario, a better understanding of the underlying mechanism in the production and functionalization of BNS based materials to overcome limitations is crucial and needs extensive theoretical and experimental understanding.

One-dimensional nanomaterials such as CNT, CNS, and BNS entail unique functionalities, which allow them to be potential candidates in in-vivo and in-vitro studies. However, the toxic nature and potential anti- or pro-bacterial actions of carbon-based materials hinder their use as biomaterials. On the contrary, hexagonal boron nitride and its derivatives show excellent biocompatibility [125-128] and could be used as biomarkers, templates for DNA self-assembly and sequencing, and carriers for targeted drug delivery. In this regard, doping with magnetic nanomaterials can be an excellent choice to guide BNS to release drugs for cancer treatment magnetically. Porous BN nanoscrolls can also be an excellent choice as water cleaning materials benefiting from their higher surface to volume ratio. Attributing to the fact that BN nanosheets, nanotubes, nanomeshes, nanoribbons and nanoflowers have many similarities of chemical and insulating behavior, it may be presumed that enhanced performance is demonstrated when BNS are used for fillers in polymer nanocomposites [129, 130]. Furthermore, BNS can also be used in polymeric biomaterials, which can ultimately result in a more extended degradation rate, and enhanced properties without any adverse effects [131, 132]. BNS also find future applications in areas of sensing devices similar to the studies carried on BNNTs [133, 134], which for instance can combine with other nanomaterials to construct novel devices for biological and medical diagnostics.

Deducing from the most up-to-date literature on BNS, it is evident that BNS possess unique properties that could potentially lead to significant evolution and growth in environmental and biological applications. For instance, despite the technological advancements made in the health sector, global diseases such as cancer remain extensive challenges for humanity, affecting developed and developing countries equally. It may be too early but not too propitious to assume that BNS might have an answer to this menacing problem. BNS with excellent biocompatibility can be made able to wrap a significant amount of drug that can be 
magnetically guided to pinpoint a specific cancerous cell, thereby irradiating the disease without affecting standard cellular functions.

Conflict of Interest.

There are no conflicts to declare.

Acknowledgments.

The authors would like to thank Mari-Ann Einarsrud for proofreading, commenting, and adding valuable suggestions and insights to the manuscript.

\section{References.}

[1] K. S. Novoselov et al., "Electric field effect in atomically thin carbon films," science, vol. 306, no. 5696, pp. 666-669, 2004.

[2] Z. Gao, C. Bumgardner, N. Song, Y. Zhang, J. Li, and X. J. N. C. Li, "Cotton-textile-enabled flexible self-sustaining power packs via roll-to-roll fabrication," Nature communications, vol. 7, p. 11586, 2016.

[3] K. Choi, Y. T. Lee, and S. J. N. T. Im, "Two-dimensional van der Waals nanosheet devices for future electronics and photonics," Nano Today, vol. 11, no. 5, pp. 626-643, 2016.

[4] J. Son et al., "Atomically precise graphene etch stops for three dimensional integrated systems from two dimensional material heterostructures," Nature communications, vol. 9, no. 1, p. $3988,2018$.

[5] Q. Li, N. Mahmood, J. Zhu, Y. Hou, and S. J. N. T. Sun, "Graphene and its composites with nanoparticles for electrochemical energy applications," Nano Today, vol. 9, no. 5, pp. 668683, 2014.

[6] C. Chung, Y.-K. Kim, D. Shin, S.-R. Ryoo, B. H. Hong, and D.-H. J. A. o. c. r. Min, "Biomedical applications of graphene and graphene oxide," Accounts of chemical research, vol. 46, no. 10, pp. 2211-2224, 2013.

[7] V. Georgakilas et al., "Noncovalent functionalization of graphene and graphene oxide for energy materials, biosensing, catalytic, and biomedical applications," Chemical reviews, vol. 116, no. 9, pp. 5464-5519, 2016.

[8] Y. Chen, C. Tan, H. Zhang, and L. J. C. S. R. Wang, "Two-dimensional graphene analogues for biomedical applications," Chemical Society Reviews, vol. 44, no. 9, pp. 2681-2701, 2015.

[9] K. Gong et al., "Reinforcing effects of graphene oxide on portland cement paste," Journal of Materials in Civil Engineering, vol. 27, no. 2, p. A4014010, 2014.

[10] D. H. Seo et al., "Anti-fouling graphene-based membranes for effective water desalination," Nature communications, vol. 9, no. 1, p. 683, 2018.

[11] A. C. Neto and K. Novoselov, "New directions in science and technology: two-dimensional crystals," Reports on Progress in Physics, vol. 74, no. 8, p. 082501, 2011.

[12] J. Carpenter. (2011, 28 June). UK invests in graphene technology. Available:

https://www.bbc.com/news/science-environment-15152609

[13] D. K. Ghaffarzadeh. (2015, 28 June). How will graphene market change in the future? Available: https://www.printedelectronicsworld.com/articles/8655/how-will-the-graphenemarket-change-in-the-future

[14] E. Gibney. (2015, November 11). The super materials that could trump graphene. Available: https://www.nature.com/news/the-super-materials-that-could-trump-graphene-1.17775 
[15] G. Cassabois, P. Valvin, and B. Gil, "Hexagonal boron nitride is an indirect bandgap semiconductor," Nature Photonics, vol. 10, no. 4, p. nphoton. 2015.277, 2016.

[16] Y. Wang et al., "High temperature thermal management with boron nitride nanosheets," Nanoscale, vol. 10, no. 1, pp. 167-173, 2018.

[17] L. H. Li and Y. Chen, "Atomically thin boron nitride: unique properties and applications," Advanced Functional Materials, vol. 26, no. 16, pp. 2594-2608, 2016.

[18] L. H. Li, J. Cervenka, K. Watanabe, T. Taniguchi, and Y. Chen, "Strong oxidation resistance of atomically thin boron nitride nanosheets," ACS nano, vol. 8, no. 2, pp. 1457-1462, 2014.

[19] Z. Liu et al., "Ultrathin high-temperature oxidation-resistant coatings of hexagonal boron nitride," Nature communications, vol. 4, p. 2541, 2013.

[20] X. Chen et al., "Preparation and electrochemical hydrogen storage of boron nitride nanotubes," The Journal of Physical Chemistry B, vol. 109, no. 23, pp. 11525-11529, 2005.

[21] A. Tokarev, E. Kjeang, M. Cannon, and D. Bessarabov, "Theoretical limit of reversible hydrogen storage capacity for pristine and oxygen-doped boron nitride," International Journal of Hydrogen Energy, vol. 41, no. 38, pp. 16984-16991, 2016.

[22] P. Fu, J. Wang, R. Jia, S. Bibi, R. I. Eglitis, and H.-X. Zhang, "Theoretical study on hydrogen storage capacity of expanded h-BN systems," Computational Materials Science, vol. 139, pp. 335-340, 2017.

[23] Q. Weng et al., "Highly water-soluble, porous, and biocompatible boron nitrides for anticancer drug delivery," ACS nano, vol. 8, no. 6, pp. 6123-6130, 2014.

[24] G. Ciofani et al., "Boron nitride nanotubes: a novel vector for targeted magnetic drug delivery," Current nanoscience, vol. 5, no. 1, pp. 33-38, 2009.

[25] X. Li et al., "Multimodal luminescent-magnetic boron nitride nanotubes@ NaGdF 4: Eu structures for cancer therapy," Chemical Communications, vol. 50, no. 33, pp. 4371-4374, 2014.

[26] X. Li, C. Zhi, N. Hanagata, M. Yamaguchi, Y. Bando, and D. Golberg, "Boron nitride nanotubes functionalized with mesoporous silica for intracellular delivery of chemotherapy drugs," Chemical Communications, vol. 49, no. 66, pp. 7337-7339, 2013.

[27] G. Ciofani, V. Raffa, A. Menciassi, and A. J. N. T. Cuschieri, "Boron nitride nanotubes: an innovative tool for nanomedicine," Nano Today, vol. 4, no. 1, pp. 8-10, 2009.

[28] J. Li, R. Dahal, S. Majety, J. Lin, and H. Jiang, "Hexagonal boron nitride epitaxial layers as neutron detector materials," Nuclear Instruments and Methods in Physics Research Section A: Accelerators, Spectrometers, Detectors and Associated Equipment, vol. 654, no. 1, pp. 417-420, 2011.

[29] T. H. Ferreira, M. C. Miranda, Z. Rocha, A. S. Leal, D. A. Gomes, and E. Sousa, "An Assessment of the Potential Use of BNNTs for Boron Neutron Capture Therapy," Nanomaterials, vol. 7, no. 4, p. 82, 2017.

[30] G. Ciofani, V. Raffa, A. Menciassi, and A. Cuschieri, "Folate functionalized boron nitride nanotubes and their selective uptake by glioblastoma multiforme cells: implications for their use as boron carriers in clinical boron neutron capture therapy," Nanoscale research letters, vol. 4, no. 2, p. 113, 2009.

[31] T. H. Ferreira et al., "Folate-grafted boron nitride nanotubes: possible exploitation in cancer therapy," International journal of pharmaceutics, vol. 481, no. 1-2, pp. 56-63, 2015.

[32] M. Nurunnabi, M. Nafiujjaman, S.-J. Lee, I.-K. Park, K. M. Huh, and Y.-k. Lee, "Preparation of ultra-thin hexagonal boron nitride nanoplates for cancer cell imaging and neurotransmitter sensing," Chemical Communications, vol. 52, no. 36, pp. 6146-6149, 2016.

[33] Y. A. Wu et al., "Utilizing boron nitride sheets as thin supports for high resolution imaging of nanocrystals," Nanotechnology, vol. 22, no. 19, p. 195603, 2011.

[34] J. Peng, S. Wang, P.-H. Zhang, L.-P. Jiang, J.-J. Shi, and J.-J. Zhu, "Fabrication of graphene quantum dots and hexagonal boron nitride nanocomposites for fluorescent cell imaging," Journal of biomedical nanotechnology, vol. 9, no. 10, pp. 1679-1685, 2013. 
[35] B. Liu et al., "One-Step Synthesis of Boron Nitride Quantum Dots: Simple Chemistry Meets Delicate Nanotechnology," Chemistry-A European Journal, vol. 22, no. 52, pp. 18899-18907, 2016.

[36] A. L. Tiano et al., "Boron nitride nanotube: synthesis and applications," in Nanosensors, Biosensors, and Info-Tech Sensors and Systems 2014, 2014, vol. 9060, p. 906006: International Society for Optics and Photonics.

[37] C. H. Lee, M. Xie, V. Kayastha, J. Wang, and Y. K. J. C. o. M. Yap, "Patterned growth of boron nitride nanotubes by catalytic chemical vapor deposition," Chemistry of Materials, vol. 22, no. 5, pp. 1782-1787, 2010.

[38] H. Zeng et al., "“White graphenes": boron nitride nanoribbons via boron nitride nanotube unwrapping," Nano letters, vol. 10, no. 12, pp. 5049-5055, 2010.

[39] X. Chen, R. A. Boulos, J. F. Dobson, and C. L. Raston, "Shear induced formation of carbon and boron nitride nano-scrolls," Nanoscale, vol. 5, no. 2, pp. 498-502, 2013.

[40] X. Li et al., "Exfoliation of hexagonal boron nitride by molten hydroxides," Advanced Materials, vol. 25, no. 15, pp. 2200-2204, 2013.

[41] Y. Wang, Z. Shi, and J. Yin, "Boron nitride nanosheets: large-scale exfoliation in methanesulfonic acid and their composites with polybenzimidazole," Journal of Materials Chemistry, vol. 21, no. 30, pp. 11371-11377, 2011.

[42] C. Ohata, R. Tagami, Y. Nakanishi, R. Iwaki, K. Nomura, and J. Haruyama, "Hexagonal boronnitride nanomesh magnets," Applied Physics Letters, vol. 109, no. 13, p. 133110, 2016.

[43] G. Lian, X. Zhang, M. Tan, S. Zhang, D. Cui, and Q. Wang, "Facile synthesis of 3D boron nitride nanoflowers composed of vertically aligned nanoflakes and fabrication of graphene-like BN by exfoliation," Journal of Materials Chemistry, vol. 21, no. 25, pp. 9201-9207, 2011.

[44] G. Carotenuto, A. Longo, S. De Nicola, C. Camerlingo, and L. Nicolais, "A simple mechanical technique to obtain carbon nanoscrolls from graphite nanoplatelets," Nanoscale research letters, vol. 8, no. 1, p. 403, 2013.

[45] M. E. Schmidt et al., "Controlled fabrication of electrically contacted carbon nanoscrolls," Nanotechnology, vol. 29, no. 23, p. 235605, 2018.

[46] X. Xie et al., "Controlled fabrication of high-quality carbon nanoscrolls from monolayer graphene," Nano letters, vol. 9, no. 7, pp. 2565-2570, 2009.

[47] D. Xia et al., "Fabrication of carbon nanoscrolls from monolayer graphene," Small, vol. 6, no. 18, pp. 2010-2019, 2010.

[48] Y. Wang and Y. Zhang, "Superior thermal conductivity of carbon nanoscroll based thermal interface materials," in Electronic Components and Technology Conference (ECTC), 2015 IEEE 65th, 2015, pp. 1234-1239: IEEE.

[49] G. W. Hanson, "Fundamental transmitting properties of carbon nanotube antennas," in Antennas and Propagation Society International Symposium, 2005 IEEE, 2005, vol. 3, pp. 247-250: IEEE.

[50] M. Hassanzadazar, M. Ahmadi, R. Ismail, and H. Goudarzi, "Electrical property analytical prediction on archimedes chiral carbon nanoscrolls," Journal of Electronic Materials, vol. 45, no. 10, pp. 5404-5411, 2016.

[51] M. Rahmani, H. Ghafoori Fard, M. T. Ahmadi, and K. Rahmani, "Analytical prediction of carbon nanoscroll-based electrochemical glucose biosensor performance," International Journal of Environmental Analytical Chemistry, vol. 97, no. 11, pp. 1024-1036, 2017.

[52] T. Zhang, Q. Xue, S. Zhang, and M. J. N. T. Dong, "Theoretical approaches to graphene and graphene-based materials," Nano Today, vol. 7, no. 3, pp. 180-200, 2012.

[53] X. Blase, A. Rubio, S. Louie, and M. Cohen, "Stability and band gap constancy of boron nitride nanotubes," EPL (Europhysics Letters), vol. 28, no. 5, p. 335, 1994.

[54] S.-H. Jhi and Y.-K. Kwon, "Hydrogen adsorption on boron nitride nanotubes: a path to roomtemperature hydrogen storage," Physical Review B, vol. 69, no. 24, p. 245407, 2004. 
[55] R. Ma, Y. Bando, H. Zhu, T. Sato, C. Xu, and D. Wu, "Hydrogen uptake in boron nitride nanotubes at room temperature," Journal of the American Chemical Society, vol. 124, no. 26, pp. 7672-7673, 2002.

[56] E. S. Permyakova et al., "Synthesis and characterization of folate conjugated boron nitride nanocarriers for targeted drug delivery," The Journal of Physical Chemistry C, vol. 121, no. 50, pp. 28096-28105, 2017.

[57] X. Li et al., "Hollow boron nitride nanospheres as boron reservoir for prostate cancer treatment," Nature communications, vol. 8, p. 13936, 2017.

[58] S. Chivilikhin, I. Y. Popov, and V. Gusarov, "Dynamics of nanotube twisting in a viscous fluid," in Doklady Physics, 2007, vol. 52, no. 1, pp. 60-62: Springer.

[59] N. Marom et al., "Stacking and registry effects in layered materials: the case of hexagonal boron nitride," Physical review letters, vol. 105, no. 4, p. 046801, 2010.

[60] D.-H. Kim, H.-S. Kim, M. W. Song, S. Lee, and S. Y. Lee, "Geometric and electronic structures of monolayer hexagonal boron nitride with multi-vacancy," Nano convergence, vol. 4, no. 1, p. 13, 2017.

[61] K. Ba et al., "Chemical and bandgap engineering in monolayer hexagonal boron nitride," Scientific Reports, vol. 7, p. 45584, 2017.

[62] J. Bao et al., "Two-dimensional hexagonal boron nitride as lateral heat spreader in electrically insulating packaging," Journal of Physics D: Applied Physics, vol. 49, no. 26, p. 265501, 2016.

[63] M. Uddin, T. Doan, J. Li, K. Ziemer, J. Lin, and H. Jiang, "Electrical transport properties of (BN)-rich hexagonal (BN) C semiconductor alloys," AIP Advances, vol. 4, no. 8, p. 087141, 2014.

[64] W. Zhou, J. Zuo, X. Zhang, and A. Zhou, "Thermal, electrical, and mechanical properties of hexagonal boron nitride-reinforced epoxy composites," Journal of Composite Materials, vol. 48, no. 20, pp. 2517-2526, 2014.

[65] E. Perim, L. D. Machado, and D. S. Galvao, "A brief review on syntheses, structures, and applications of nanoscrolls," Frontiers in Materials, vol. 1, p. 31, 2014.

[66] S. Chivilikhin, I. Y. Popov, A. Svitenkov, D. Chivilikhin, and V. Gusarov, "Formation and evolution of nanoscroll ensembles based on layered-structure compounds," in Doklady Physics, 2009, vol. 54, no. 11, p. 491: Springer.

[67] E. Perim and D. S. Galvao, "The structure and dynamics of boron nitride nanoscrolls," Nanotechnology, vol. 20, no. 33, p. 335702, 2009.

[68] S. Chang, C. Chiang, and S. Lin, "Feature-rich Geometric and Electronic Properties of Carbon Nanoscrolls," arXiv preprint arXiv:1607.00452, 2016.

[69] C. Yan, J. Liu, F. Liu, J. Wu, K. Gao, and D. Xue, "Tube formation in nanoscale materials," Nanoscale research letters, vol. 3, no. 12, p. 473, 2008.

[70] S. F. Braga, V. R. Coluci, S. B. Legoas, R. Giro, D. S. Galvão, and R. H. Baughman, "Structure and dynamics of carbon nanoscrolls," Nano letters, vol. 4, no. 5, pp. 881-884, 2004.

[71] A. I. Siahlo et al., "Structure and energetics of carbon, hexagonal boron nitride, and carbon/hexagonal boron nitride single-layer and bilayer nanoscrolls," Physical Review Materials, vol. 2, no. 3, p. 036001, 2018.

[72] Z. Liu, J. Gao, G. Zhang, Y. Cheng, and Y.-W. Zhang, "From two-dimensional nano-sheets to roll-up structures: expanding the family of nanoscroll," Nanotechnology, vol. 28, no. 38, p. 385704, 2017.

[73] D. S. E. G. Perim, "Boron Nitride Nanoscrolls," in Physicæ Proceedings, march. 2012.

[74] E. Perim, R. Paupitz, and D. S. Galvao, "Controlled route to the fabrication of carbon and boron nitride nanoscrolls: A molecular dynamics investigation," Journal of Applied Physics, vol. 113, no. 5, p. 054306, 2013.

[75] Y. Li, "Boron-nitride nanotube triggered self-assembly of hexagonal boron-nitride nanostructure," Physical Chemistry Chemical Physics, vol. 16, no. 38, pp. 20689-20696, 2014. 
[76] D. H. Suh, "Formation of hexagonal boron nitride nanoscrolls induced by inclusion and exclusion of self-assembling molecules in solution process," Nanoscale, vol. 6, no. 11, pp. 5686-5690, 2014.

[77] K. H. Choi, J. E. Park, and D. H. Suh, "Evolution of magnetism by rolling up hexagonal boron nitride nanosheets tailored with superparamagnetic nanoparticles," Physical Chemistry Chemical Physics, vol. 19, no. 5, pp. 4048-4055, 2017.

[78] L. Yuan et al., "A reliable way of mechanical exfoliation of large scale two dimensional materials with high quality," AIP Advances, vol. 6, no. 12, p. 125201, 2016.

[79] Y. Hernandez et al., "High-yield production of graphene by liquid-phase exfoliation of graphite," Nature nanotechnology, vol. 3, no. 9, p. 563, 2008.

[80] Y.-Z. Wang, T. Chen, X.-F. Gao, H.-H. Liu, and X.-X. Zhang, "Liquid phase exfoliation of graphite into few-layer graphene by sonication and microfluidization," Materials Express, vol. 7, no. 6, pp. 491-499, 2017.

[81] B. Tang, X. Yun, Z. Xiong, and X. Wang, "Formation of Graphene Oxide Nanoscrolls in Organic Solvents: Toward Scalable Device Fabrication," ACS Applied Nano Materials, vol. 1, no. 2, pp. 686-697, 2018.

[82] T. Fan et al., "Fabrication of high-quality graphene oxide nanoscrolls and application in supercapacitor," Nanoscale research letters, vol. 10, no. 1, p. 192, 2015.

[83] L. M. Viculis, J. J. Mack, and R. B. Kaner, "A chemical route to carbon nanoscrolls," Science, vol. 299, no. 5611, pp. 1361-1361, 2003.

[84] Z. Ding et al., "Lithium intercalation and exfoliation of layered bismuth selenide and bismuth telluride," Journal of Materials Chemistry, vol. 19, no. 17, pp. 2588-2592, 2009.

[85] X. Fan et al., "Fast and efficient preparation of exfoliated 2H MoS2 nanosheets by sonication-assisted lithium intercalation and infrared laser-induced $1 \mathrm{~T}$ to $2 \mathrm{H}$ phase reversion," Nano letters, vol. 15, no. 9, pp. 5956-5960, 2015.

[86] Q. Li et al., "Intermediate phases in sodium intercalation into MoS2 nanosheets and their implications for sodium-ion batteries," Nano Energy, vol. 38, pp. 342-349, 2017.

[87] E. Ziambaras, J. Kleis, E. Schröder, and P. Hyldgaard, "Potassium intercalation in graphite: A van der Waals density-functional study," Physical Review B, vol. 76, no. 15, p. 155425, 2007.

[88] X. Chen, L. Li, X. Sun, H. G. Kia, and H. Peng, "A novel synthesis of graphene nanoscrolls with tunable dimension at a large scale," Nanotechnology, vol. 23, no. 5, p. 055603, 2012.

[89] A. Ismach et al., "Toward the controlled synthesis of hexagonal boron nitride films," ACS Nano, vol. 6, no. 7, pp. 6378-6385, 2012.

[90] K. K. Kim et al., "Synthesis and characterization of hexagonal boron nitride film as a dielectric layer for graphene devices," ACS nano, vol. 6, no. 10, pp. 8583-8590, 2012.

[91] C.-W. Chang, W.-Q. Han, and A. Zettl, "Thermal conductivity of BCN and BN nanotubes," Journal of Vacuum Science \& Technology B: Microelectronics and Nanometer Structures Processing, Measurement, and Phenomena, vol. 23, no. 5, pp. 1883-1886, 2005.

[92] H. Şahin et al., "Monolayer honeycomb structures of group-IV elements and III-V binary compounds: First-principles calculations," Physical Review B, vol. 80, no. 15, p. 155453, 10/28/ 2009.

[93] Y. Lin and J. W. Connell, "Advances in 2D boron nitride nanostructures: nanosheets, nanoribbons, nanomeshes, and hybrids with graphene," Nanoscale, 10.1039/C2NR32201C vol. 4, no. 22, pp. 6908-6939, 2012.

[94] L. Lindsay and D. Broido, "Enhanced thermal conductivity and isotope effect in single-layer hexagonal boron nitride," Physical Review B, vol. 84, no. 15, p. 155421, 2011.

[95] L. Lindsay and D. Broido, "Theory of thermal transport in multilayer hexagonal boron nitride and nanotubes," Physical Review B, vol. 85, no. 3, p. 035436, 2012.

[96] S. M. Kim et al., "Synthesis of large-area multilayer hexagonal boron nitride for high material performance," Nature communications, vol. 6, p. 8662, 2015. 
[97] C. F. Woellner, L. D. Machado, P. A. S. Autreto, J. M. de Sousa, and D. S. Galvao, "Structural transformations of carbon and boron nitride nanoscrolls at high impact collisions," Physical Chemistry Chemical Physics, 10.1039/C7CP07402F vol. 20, no. 7, pp. 4911-4916, 2018.

[98] Y. Li, W. Zhang, B. Guo, and D. Datta, "Interlayer shear of nanomaterials: Graphenegraphene, boron nitride-boron nitride and graphene-boron nitride," acta mechanica solida sinica, vol. 30, no. 3, pp. 234-240, 2017.

[99] J. Wang et al., "Flower stamen-like porous boron carbon nitride nanoscrolls for water cleaning," Nanoscale, vol. 9, no. 28, pp. 9787-9791, 2017.

[100] M. H. Tahersima and V. J. Sorger, "Enhanced photon absorption in spiral nanostructured solar cells using layered 2D materials," Nanotechnology, vol. 26, no. 34, p. 344005, 2015.

[101] A. L. M. Reddy, A. E. Tanur, and G. C. Walker, "Synthesis and hydrogen storage properties of different types of boron nitride nanostructures," international journal of hydrogen energy, vol. 35, no. 9, pp. 4138-4143, 2010.

[102] T. Li, M. Lin, Y. Huang, and T. Lin, "Quantum transport in carbon nanoscrolls," Physics letters $A$, vol. 376, no. 4, pp. 515-520, 2012.

[103] J. Zheng et al., "Production of High-Quality Carbon Nanoscrolls with Microwave Spark Assistance in Liquid Nitrogen," Advanced materials, vol. 23, no. 21, pp. 2460-2463, 2011.

[104] L. Jiang, L. Gao, and J. Sun, "Production of aqueous colloidal dispersions of carbon nanotubes," Journal of colloid and interface science, vol. 260, no. 1, pp. 89-94, 2003.

[105] D. Golberg, "Synthesis and characterization of ropes made of BN multiwalled nanotubes," Scripta Materialia, vol. 44, no. 8, pp. 1561-1565, 2001.

[106] Y. Chen, J. Zou, S. J. Campbell, and G. Le Caer, "Boron nitride nanotubes: pronounced resistance to oxidation," Applied physics letters, vol. 84, no. 13, pp. 2430-2432, 2004.

[107] C. Zhi, Y. Bando, C. Tang, S. Honda, H. Kuwahara, and D. Golberg, "Boron nitride nanotubes/polystyrene composites," Journal of Materials Research, vol. 21, no. 11, pp. 2794-2800, 2006.

[108] X. Chen et al., "Boron nitride nanotubes are noncytotoxic and can be functionalized for interaction with proteins and cells," Journal of the American Chemical Society, vol. 131, no. 3, pp. 890-891, 2009.

[109] L. Horvath et al., "In vitro investigation of the cellular toxicity of boron nitride nanotubes," ACS nano, vol. 5, no. 5, pp. 3800-3810, 2011.

[110] N. Wang et al., "Toxicity evaluation of boron nitride nanospheres and water-soluble boron nitride in Caenorhabditis elegans," International journal of nanomedicine, vol. 12, p. 5941, 2017.

[111] M. Rasel, T. Li, T. Nguyen, and Y. Gu, "The Assessment of Toxicity of Boron Nitride Nanoparticle Using Atomic Forced Microscopy," in 7th WACBE World Congress on Bioengineering 2015, 2015, pp. 31-34: Springer.

[112] G. Ciofani, S. Danti, G. G. Genchi, B. Mazzolai, and V. Mattoli, "Boron nitride nanotubes: biocompatibility and potential spill-over in nanomedicine," Small, vol. 9, no. 9-10, pp. 16721685, 2013.

[113] V. K. Kodali et al., "Acute in vitro and in vivo toxicity of a commercial grade boron nitride nanotube mixture," Nanotoxicology, vol. 11, no. 8, pp. 1040-1058, 2017.

[114] Y. Volkov, J. McIntyre, and A. Prina-Mello, "Graphene toxicity as a double-edged sword of risks and exploitable opportunities: a critical analysis of the most recent trends and developments," 2D Materials, vol. 4, no. 2, p. 022001, 2017.

[115] T. A. Tabish, S. Zhang, and P. G. Winyard, "Developing the next generation of graphenebased platforms for cancer therapeutics: the potential role of reactive oxygen species," Redox biology, 2017.

[116] T. A. Tabish et al., "Investigation into the toxic effects of graphene nanopores on lung cancer cells and biological tissues," Applied Materials Today, vol. 12, pp. 389-401, 2018. 
[117] A. B. Seabra, A. J. Paula, R. de Lima, O. L. Alves, and N. Durán, "Nanotoxicity of graphene and graphene oxide," Chemical research in toxicology, vol. 27, no. 2, pp. 159-168, 2014.

[118] C. Fisher, A. E. Rider, Z. J. Han, S. Kumar, I. Levchenko, and K. Ostrikov, "Applications and nanotoxicity of carbon nanotubes and graphene in biomedicine," Journal of Nanomaterials, vol. 2012, p. 3, 2012.

[119] L. Ma-Hock et al., "Comparative inhalation toxicity of multi-wall carbon nanotubes, graphene, graphite nanoplatelets and low surface carbon black," Particle and fibre toxicology, vol. 10, no. 1, p. 23, 2013.

\{120] Matharu, Rupy Kaur, et al. "The effect of graphene-poly (methyl methacrylate) fibres on microbial growth." Interface focus 8.3 (2018): 20170058.

[121] Heseltine, Phoebe L., Jubair Ahmed, and Mohan Edirisinghe. "Developments in pressurized gyration for the mass production of polymeric fibers." Macromolecular Materials and Engineering 303.9 (2018): 1800218.

[122] Tabish, Tanveer A., et al. "Influence of luminescent graphene quantum dots on trypsin activity." International Journal of Nanomedicine 13 (2018): 1525.

[123] Matharu, Rupy Kaur, Lena Ciric, and Mohan Edirisinghe. "Nanocomposites: suitable alternatives as antimicrobial agents." Nanotechnology 29.28 (2018): 282001

[124] E. P. Randviir, D. A. Brownson, and C. E. Banks, "A decade of graphene research: production, applications and outlook," Materials Today, vol. 17, no. 9, pp. 426-432, 2014.

[125] L. Jing et al., "Biocompatible hydroxylated boron nitride nanosheets/poly (vinyl alcohol) interpenetrating hydrogels with enhanced mechanical and thermal responses," ACS nano, vol. 11, no. 4, pp. 3742-3751, 2017.

[126] A. Salvetti et al., "In vivo biocompatibility of boron nitride nanotubes: effects on stem cell biology and tissue regeneration in planarians," Nanomedicine, vol. 10, no. 12, pp. 19111922, 2015.

[127] S. Feng et al., "Folate-conjugated boron nitride nanospheres for targeted delivery of anticancer drugs," International journal of nanomedicine, vol. 11, p. 4573, 2016.

[128] T. H. Ferreira, P. Silva, R. Santos, and E. Sousa, "A novel synthesis route to produce boron nitride nanotubes for bioapplications," Journal of biomaterials and nanobiotechnology, vol. 2, no. 04, p. 426, 2011.

[129] A. Pullanchiyodan, K. S. Nair, and K. P. Surendran, "Silver-Decorated Boron Nitride Nanosheets as an Effective Hybrid Filler in PMMA for High-Thermal-Conductivity Electronic Substrates," ACS Omega, vol. 2, no. 12, pp. 8825-8835, 2017.

[130] T. Wang et al., "Enhanced Thermal Conductivity of Polyimide Composites with Boron Nitride Nanosheets," Scientific reports, vol. 8, no. 1, p. 1557, 2018.

[131] D. Lahiri et al., "Boron nitride nanotube reinforced polylactide-polycaprolactone copolymer composite: mechanical properties and cytocompatibility with osteoblasts and macrophages in vitro," Acta biomaterialia, vol. 6, no. 9, pp. 3524-3533, 2010.

[132] D. Lahiri, V. Singh, A. P. Benaduce, S. Seal, L. Kos, and A. Agarwal, "Boron nitride nanotube reinforced hydroxyapatite composite: mechanical and tribological performance and in-vitro biocompatibility to osteoblasts," Journal of the mechanical behavior of biomedical materials, vol. 4, no. 1, pp. 44-56, 2011.

[133] A. L. M. Reddy, B. K. Gupta, T. N. Narayanan, A. A. Martí, P. M. Ajayan, and G. C. Walker, "Probing of Ni-encapsulated ferromagnetic boron nitride nanotubes by time-resolved and steady-state photoluminescence spectroscopy," The Journal of Physical Chemistry $C$, vol. 116, no. 23, pp. 12803-12809, 2012.

[134] Y. Yu, H. Chen, Y. Liu, L. H. Li, and Y. Chen, "Humidity sensing properties of single Audecorated boron nitride nanotubes," Electrochemistry communications, vol. 30, pp. 29-33, 2013. 